\title{
Observation of the $B_{s}^{0} \rightarrow J / \psi \phi \phi$ decay
}

\section{KHCh}

\section{The LHCb collaboration}

\section{E-mail: emanuelesantovetti@gmail.com}

ABSTRACT: The $B_{s}^{0} \rightarrow J / \psi \phi \phi$ decay is observed in $p p$ collision data corresponding to an integrated luminosity of $3 \mathrm{fb}^{-1}$ recorded by the $\mathrm{LHCb}$ detector at centre-of-mass energies of $7 \mathrm{TeV}$ and $8 \mathrm{TeV}$. This is the first observation of this decay channel, with a statistical significance of 15 standard deviations. The mass of the $B_{s}^{0}$ meson is measured to be $5367.08 \pm 0.38 \pm 0.15 \mathrm{MeV} / \mathrm{c}^{2}$. The branching fraction ratio $\mathcal{B}\left(B_{s}^{0} \rightarrow J / \psi \phi \phi\right) / \mathcal{B}\left(B_{s}^{0} \rightarrow\right.$ $J / \psi \phi)$ is measured to be $0.0115 \pm 0.0012{ }_{-0.0009}^{+0.0005}$. In both cases, the first uncertainty is statistical and the second is systematic. No evidence for non-resonant $B_{s}^{0} \rightarrow J / \psi \phi K^{+} K^{-}$ or $B_{s}^{0} \rightarrow J / \psi K^{+} K^{-} K^{+} K^{-}$decays is found.

KEYwords: B physics, Branching fraction, Hadron-Hadron scattering, QCD, Spectroscopy

ARXIV EPRINT: 1601.05284 


\section{Contents}

1 Introduction $\quad 1$

2 The LHCb detector 2

3 Event selection 3

4 Results 4

5 Systematic uncertainties $\quad 6$

6 Conclusions $\quad 9$

$\begin{array}{ll}\text { The LHCb collaboration } & 13\end{array}$

\section{Introduction}

The study of the $B_{s}^{0} \rightarrow J / \psi \phi \phi$ decay, previously unobserved, allows a precise measurement of the $B_{s}^{0}$ meson mass and a search for possible resonances in the $\phi \phi$ and $J / \psi \phi$ invariant mass spectra, similar to what has been reported for the $B^{+} \rightarrow J / \psi \phi K^{+}$decay mode [13] (the inclusion of charge conjugate processes is implied throughout). The most recent theoretical predictions for heavy hadron masses, based on lattice QCD calculations, can be found in refs. [4-6]. The current experimental knowledge of the $B_{s}^{0}$ mass, as summarized in ref. [7], is dominated by results from the LHCb experiment [8], which were obtained with the $B_{s}^{0} \rightarrow J / \psi \phi$ decay using a small fraction of the integrated luminosity collected in the 2010-2012 LHC run. The $B_{s}^{0}$ mass measurement using this decay is limited by the precision of the momentum scale. The $B_{s}^{0} \rightarrow J / \psi \phi \phi$ decay mode is a good alternative to $B_{s}^{0} \rightarrow J / \psi \phi$ since the kinetic energy available to the final-state particles ( $Q$-value) is much lower, leading to a $65 \%$ reduction in the systematic uncertainty arising from the precision of the momentum scale.

The $B_{s}^{0} \rightarrow J / \psi \phi \phi$ decay is also of interest in searches for intermediate states in the $B_{s}^{0}$ decay chain. In recent years, many new charmonium or charmonium-like states have been discovered, which are not easily accommodated in the quark model of hadrons $[9,10]$. In a study of $B^{+} \rightarrow J / \psi \phi K^{+}$decays, the CDF collaboration reported evidence for a state, in the $J / \psi \phi$ invariant mass spectrum, called $Y(4140)$ with mass and width values of $m=4143.0 \pm 2.9$ (stat) \pm 1.2 (syst) $\mathrm{MeV} / c^{2}$ and $\Gamma=11.7_{-5.0}^{+8.3}$ (stat) \pm 3.7 (syst) $\mathrm{MeV}$ [3]. The Belle and BaBar collaborations searched for the $Y(4140)$ using the same $B^{+}$decay mode $[1,11]$ and found no significant signal, although the upper limits on the production rate did not contradict the CDF measurement. Recently, the D0 collaboration reported a similar structure [12]. At the LHC, both the LHCb and CMS collaborations have searched 
for the state in question. The LHCb collaboration found no evidence with $0.37 \mathrm{fb}^{-1}$ of $p p$ collision data [2], in 2.4 $\sigma$ disagreement with the CDF measurement. A CMS search for the same signature [13] supports the CDF observation. With two out of five experiments failing to observe the $Y(4140)$ resonance the question of its existence still remains open. The search for resonances in the $\phi \phi$ invariant mass spectrum is also of interest. Several experiments have reported a near-threshold enhancement in the $\phi \phi$ invariant mass distribution from the $J / \psi \rightarrow \gamma \phi \phi$ decay [14-16]. A partial-wave analysis showed that the structure is dominated by a $0^{-+}$state called $\eta(2225)$. This resonance is still controversial and its observation in a different decay mode would be conclusive.

Theoretical predictions of the $B_{s}^{0} \rightarrow J / \psi \phi \phi$ branching fraction are difficult due to the presence of three vector mesons in the final states. The $B_{s}^{0} \rightarrow J / \psi \phi \phi$ decay is the $B_{s}^{0}$ counterpart of the measured $B^{+} \rightarrow J / \psi \phi K^{+}$and $B^{0} \rightarrow J / \psi \phi K^{0}$ decays [17]. All these channels are strongly suppressed with respect to the similar decays without the additional $\phi$ meson in the final state. The suppression factors of the last two channels are $0.048 \pm 0.004$ and $0.057 \pm 0.012$ for the charged and neutral decays [7]. A qualitative comparison with these branching fractions can be done considering that the phase space of the decay $B_{s}^{0} \rightarrow J / \psi \phi \phi$ is smaller by a factor of seven, so the $B_{s}^{0} \rightarrow J / \psi \phi \phi$ branching fraction is expected to be $\sim 10^{-5}$.

This paper presents the first observation of the decay $B_{s}^{0} \rightarrow J / \psi \phi \phi$ and the decay branching fraction measurement with respect to the reference decay $B_{s}^{0} \rightarrow J / \psi \phi$. A measurement of the $B_{s}^{0}$ mass is also presented. The data sample corresponds to an integrated lu-

minosity of $3.0 \mathrm{fb}^{-1} p p$ collisions collected by the $\mathrm{LHCb}$ experiment. The data were recorded in the years 2011 and 2012 at centre-of-mass energies of $7 \mathrm{TeV}$ and $8 \mathrm{TeV}$, respectively.

\section{The $\mathrm{LHCb}$ detector}

The LHCb detector $[18,19]$ is a single-arm forward spectrometer covering the pseudorapidity range $2<\eta<5$, designed for the study of particles containing $b$ or $c$ quarks. The detector includes a high-precision tracking system consisting of a silicon-strip vertex detector surrounding the $p p$ interaction region, a large-area silicon-strip detector located upstream of a dipole magnet with a bending power of about $4 \mathrm{Tm}$, and three stations of silicon-strip detectors and straw drift tubes placed downstream of the magnet. The polarity of the dipole magnet is reversed periodically throughout data-taking. The tracking system provides a measurement of momentum, $p$, of charged particles with a relative uncertainty that varies from $0.5 \%$ at low momentum to $1.0 \%$ at $200 \mathrm{GeV} / c$. The minimum distance of a track to a primary vertex (PV), the impact parameter, is measured with a resolution of $\left(15+29 / p_{\mathrm{T}}\right) \mu \mathrm{m}$, where $p_{\mathrm{T}}$ is the component of the momentum transverse to the beam, in $\mathrm{GeV} / c$. Different types of charged hadrons are distinguished using information from two ring-imaging Cherenkov detectors. Photons, electrons and hadrons are identified by a calorimeter system consisting of scintillating-pad and preshower detectors, an electromagnetic calorimeter and a hadronic calorimeter. Muons are identified by a system composed of alternating layers of iron and multiwire proportional chambers. The online event selection is performed by a trigger, which consists of a hardware stage, based on 
information from the calorimeter and muon systems, followed by a software stage, which applies a full event reconstruction.

Simulated events are used to determine trigger, reconstruction and selection efficiencies and reconstructed mass distributions. In addition, simulated samples are used to estimate possible peaking backgrounds from $B$ meson decays that can mimic the $B_{s}^{0} \rightarrow J / \psi \phi(\phi)$ final states. In the simulation, $p p$ collisions are generated using PүтнIA 6 [20] with a specific LHCb configuration [21]. Decays of hadronic particles are described by EvTGEN [22], in which final-state radiation is generated using Pнотоs [23]. The interaction of the generated particles with the detector, and its response, are implemented using the GEANT4 toolkit $[24,25]$ as described in ref. [26].

\section{Event selection}

The final states of the signal and reference channels differ only by the presence of an extra $\phi$ meson in the former mode. The selections of the $B_{s}^{0} \rightarrow J / \psi \phi \phi$ and $B_{s}^{0} \rightarrow J / \psi \phi$ candidates are done in almost the same way, allowing a partial cancellation of systematic uncertainties in the evaluation of the efficiency ratio. The $J / \psi$ meson is reconstructed in the $J / \psi \rightarrow \mu^{+} \mu^{-}$decay while the $\phi$ meson is reconstructed in the $\phi \rightarrow K^{+} K^{-}$decay.

Events are selected by the hardware triggers requiring a single muon with transverse momentum $p_{\mathrm{T}}>1.48 \mathrm{GeV} / c$ or a muon pair with product of transverse momenta greater than $(1.3 \mathrm{GeV} / c)^{2}$. At the first stage of the software trigger, events are selected that contain two muon tracks with $p_{\mathrm{T}}>0.5 \mathrm{GeV} / \mathrm{c}$ and invariant mass $m\left(\mu^{+} \mu^{-}\right)>2.7 \mathrm{GeV} / c^{2}$, or a single muon track with $p_{\mathrm{T}}>1 \mathrm{GeV} / c$ and $\chi_{\mathrm{IP}}^{2}>16$ with respect to any PV. The quantity $\chi_{\mathrm{IP}}^{2}$ is the difference between the $\chi^{2}$ values of a given PV reconstructed with and without the track considered. The second stage of the software trigger selects a muon pair with an invariant mass that is consistent with the known $J / \psi$ mass [7]. The decay length significance of the reconstructed $J / \psi$ candidate, $S_{L}$, is required to be greater than 3 , where $S_{L}$ is the distance between the $J / \psi$ vertex and the PV, divided by its uncertainty.

The offline analysis uses a cut-based preselection, followed by a multivariate analysis. In the preselection all the tracks are required to have a good-quality track fit. In the $\phi \rightarrow$ $K^{+} K^{-}$decay reconstruction, kaons are selected with $p>3 \mathrm{GeV} / c$ and $p_{\mathrm{T}}>200 \mathrm{MeV} / c$, and the vertex is required to have a good-quality fit. Particle identification (PID) is performed using information from all the subdetectors. A loose requirement is applied to the PID discriminant of kaons with respect to the pion misidentification DLL $_{K \pi}>0$, where $\mathrm{DLL}_{x \pi}=\ln \mathcal{L}_{x}-\ln \mathcal{L}_{\pi}$ is the delta-log-likelihood for the $x$ particle hypothesis with respect to the pion. For the $J / \psi \rightarrow \mu^{+} \mu^{-}$decay, the two muons are required to have $p>5 \mathrm{GeV} / c$ and to satisfy a loose PID selection, DLL $_{\mu \pi}>-1$. The invariant mass of the $J / \psi$ candidate is required to be in the interval $[3036,3156] \mathrm{MeV} / c^{2}$, corresponding to a $\pm 4 \sigma$ interval around the nominal mass of the $J / \psi$ meson [7]. To select the final $B_{s}^{0} \rightarrow J / \psi \phi(\phi)$ decay, the $\phi$ and $J / \psi$ meson candidates are required to pass the selection cuts $p_{\mathrm{T}}(\phi)>300 \mathrm{MeV} / c$ and $p_{\mathrm{T}}(J / \psi)>400 \mathrm{MeV} / c$, and to form a good-quality displaced vertex. The collinearity angle, defined as the angle between the reconstructed $B_{s}^{0}$ momentum and the flight direction determined from the secondary vertex, is required to be smaller than $1.8^{\circ}$. In the $B_{s}^{0} \rightarrow$ 
$J / \psi \phi$ decay selection, to reduce the contamination from non-resonant $B_{s}^{0} \rightarrow J / \psi K^{+} K^{-}$ decays, the dikaon invariant mass is required to be in the range $[980,1080] \mathrm{MeV} / c^{2}$. To improve the mass and decay-time resolutions, a kinematic fit [27] is applied to both $B_{s}^{0}$ decays, constraining the mass of the $J / \psi$ candidate to its known value [7] and the $B_{s}^{0}$ momentum to point to the PV. Finally, the $B_{s}^{0}$ candidate invariant mass is required to be in the interval $[5250,5490] \mathrm{MeV} / \mathrm{c}^{2}$.

Different multivariate selection algorithms, based on a boosted decision tree (BDT) $[28$, 29 ] with the AdaBoost algorithm [30], are used to select the signal and the reference channel samples. The BDT is trained with simulated $B_{s}^{0}$ samples for the signals, while for the background, a sample of 40 millions simulated events containing inclusive $B \rightarrow J / \psi X$ decays is used. For the $B_{s}^{0} \rightarrow J / \psi \phi \phi$ decay channel, the simulated sample is generated according to phase space. The BDT input variables are the $p_{\mathrm{T}}$ of the $\phi$ and $J / \psi$ mesons and the vertex $\chi^{2}$, flight distance significance, $S_{L}$, collinearity angle and the impact parameter of the $B_{s}^{0}$ meson with respect to the PV. The BDT discriminant threshold is chosen to maximise the figure of merit, $\epsilon /(3 / 2+\sqrt{b})$ [31], where $\epsilon$ is the signal efficiency determined using simulated events and $b$ is the number of expected background candidates estimated using mass sideband events in the data. For the $B_{s}^{0} \rightarrow J / \psi \phi$ channel the BDT discriminant is selected to maximize $s / \sqrt{s+b}$, where $s$ and $b$ are the expected signal and background yields, estimated from simulated events and sideband data, respectively.

In the $B_{s}^{0} \rightarrow J / \psi \phi \phi$ selection, no restriction is initially put on the $K^{+} K^{-}$system invariant mass, with both the resonant and the non-resonant $B_{s}^{0} \rightarrow J / \psi\left(K^{+} K^{-}\right)\left(K^{+} K^{-}\right)$ selected. If the candidate $B_{s}^{0} \rightarrow J / \psi\left(K_{1}^{+} K_{1}^{-}\right)\left(K_{2}^{+} K_{2}^{-}\right)$passes the selection cuts, almost always the candidate $B_{s}^{0} \rightarrow J / \psi\left(K_{1}^{+} K_{2}^{-}\right)\left(K_{2}^{+} K_{1}^{-}\right)$also passes the cuts, resulting in a duplicated candidate. So a genuine resonant $B_{s}^{0} \rightarrow J / \psi \phi\left(K^{+} K^{-}\right) \phi\left(K^{+} K^{-}\right)$event will most of the time produce also a "fake" non-resonant candidate, given the low probability that the invariant mass of two wrongly-coupled kaons is around the $\phi$ mass. In order to remove these "fake" candidates, the $K^{+} K^{-}$system masses are required to satisfy $\left|m\left(K^{+} K^{-}\right)-m_{\phi}\right|<15 \mathrm{MeV} / c^{2}$. After this cut, $1.8 \%$ of events contain double candidates. For each of these events, one candidate is chosen at random. In the $B_{s}^{0} \rightarrow J / \psi \phi$ decay selection, this ambiguity problem is not present, so a tight cut on the $\left|m\left(K^{+} K^{-}\right)-m_{\phi}\right|$ is not applied.

\section{Results}

The $B_{s}^{0} \rightarrow J / \psi \phi \phi$ decay branching fraction is measured with respect to the reference decay $B_{s}^{0} \rightarrow J / \psi \phi$ as

$$
\frac{\mathcal{B}\left(B_{s}^{0} \rightarrow J / \psi \phi \phi\right)}{\mathcal{B}\left(B_{s}^{0} \rightarrow J / \psi \phi\right)}=\frac{N_{\mathrm{obs}}\left(B_{s}^{0} \rightarrow J / \psi \phi \phi\right)}{\epsilon\left(B_{s}^{0} \rightarrow J / \psi \phi \phi\right)} \cdot \frac{\epsilon\left(B_{s}^{0} \rightarrow J / \psi \phi\right)}{N_{\mathrm{obs}}\left(B_{s}^{0} \rightarrow J / \psi \phi\right)} \cdot \frac{1}{\mathcal{B}\left(\phi \rightarrow K^{+} K^{-}\right)},
$$

where $N_{\mathrm{obs}}\left(B_{s}^{0} \rightarrow J / \psi \phi \phi\right)$ and $N_{\mathrm{obs}}\left(B_{s}^{0} \rightarrow J / \psi \phi\right)$ are the numbers of observed events and $\epsilon\left(B_{s}^{0} \rightarrow J / \psi \phi \phi\right)$ and $\epsilon\left(B_{s}^{0} \rightarrow J / \psi \phi\right)$ are the selection efficiencies.

Figure 1 shows the invariant mass of the reconstructed $B_{s}^{0} \rightarrow J / \psi \phi \phi$ decay, for all the candidates surviving the pre-selection, the BDT and the selection on the $m\left(K^{+} K^{-}\right)$ 


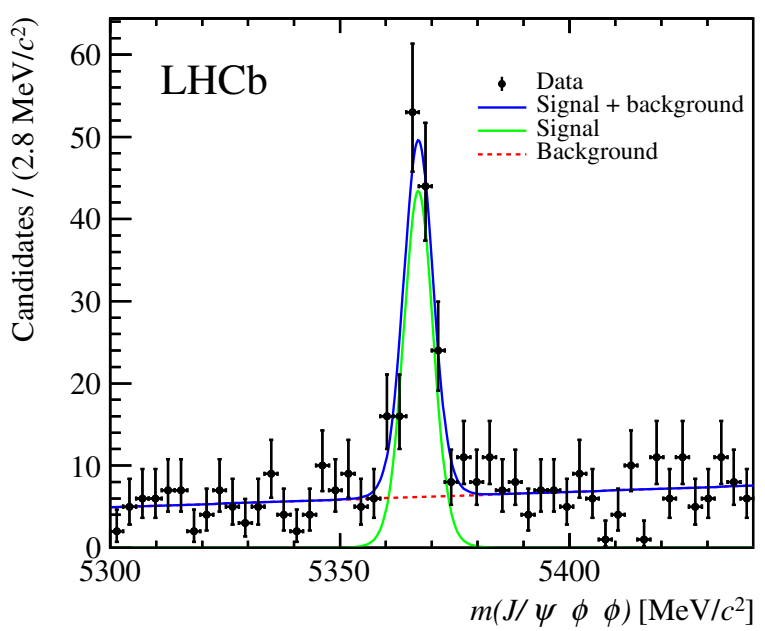

Figure 1. Invariant mass of reconstructed $B_{s}^{0} \rightarrow J / \psi \phi \phi$ candidates. The result of the fit to the distribution is also shown.

around the $\phi$ mass. In order to evaluate the number of signal decays, an unbinned extended maximum likelihood fit is performed assuming a Gaussian signal peak and an exponential combinatorial background. The observed signal yield is $128 \pm 13$ events, where the uncertainty is statistical. Using Wilks's theorem [32], the statistical significance is found to be 15 standard deviations. As expected, the mass resolution is good, $\sigma=3.05 \pm 0.41 \mathrm{MeV} / c^{2}$, due to the low $Q$-value of the decay.

In the reference channel, in order to discriminate between the resonant $B_{s}^{0} \rightarrow J / \psi \phi$ and the non-resonant $B_{s}^{0} \rightarrow J / \psi K^{+} K^{-}$decays, a fit to the $K^{+} K^{-}$mass spectrum is made, where the combinatorial background in the $B_{s}^{0}$ mass window is statistically removed using the sPlot technique [33]. Figure 2 (left) shows the mass distribution of the $B_{s}^{0} \rightarrow$ $J / \psi K^{+} K^{-}$candidates with the fit results superimposed. The $B_{s}^{0}$ peak is described by a double Crystal Ball function [34], while the underlying combinatorial background is described by an exponential function plus a second-order polynomial. Figure 2 (right) shows the $K^{+} K^{-}$mass distribution superimposed with the result of the fit. The fit is performed using a relativistic Breit-Wigner convolved with a Gaussian resolution function for the $\phi$ signal and a second-order polynomial for the non-resonant component. The observed $B_{s}^{0} \rightarrow J / \psi \phi$ yield is $82120 \pm 330$ events where the uncertainty is statistical.

All the efficiencies (detector acceptance, reconstruction, trigger and selection) are evaluated using the simulated samples together with a data-driven method [35] for tracking and PID. To check the reliability of the simulation, a comparison is made between data and simulation for all of the kinematic variables used in the selection; good agreement is found. Since the ratio of $\epsilon\left(B_{s}^{0} \rightarrow J / \psi \phi \phi\right)$ over $\epsilon\left(B_{s}^{0} \rightarrow J / \psi \phi\right)$ is evaluated, many systematic effects, related to possible small deviation of simulation with respect to data, cancel or are significantly reduced.

The efficiency ratio $\epsilon\left(B_{s}^{0} \rightarrow J / \psi \phi \phi\right) / \epsilon\left(B_{s}^{0} \rightarrow J / \psi \phi\right)$ is evaluated to be $0.2778 \pm 0.0015$, where the uncertainty is statistical, due to the limited simulated sample sizes. As expected, the efficiency of the $B_{s}^{0} \rightarrow J / \psi \phi \phi$ channel is lower than that of $B_{s}^{0} \rightarrow J / \psi \phi$ one, due to 

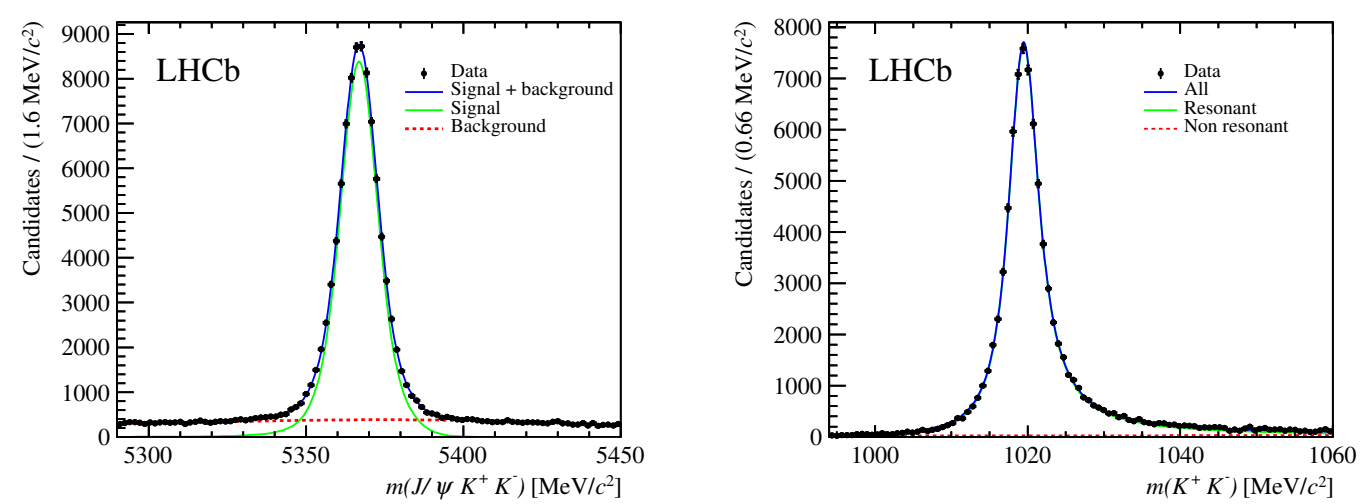

Figure 2. (Left) Invariant mass distribution of reconstructed $B_{s}^{0} \rightarrow J / \psi K^{+} K^{-}$candidates; (right) invariant mass distribution of the $K^{+} K^{-}$system, for those candidates which come from a $B_{s}^{0}$ decay. For both distributions, the fit result is shown.

the presence of the additional $\phi \rightarrow K^{+} K^{-}$decay and the fact that on average the decay products have a smaller transverse momentum.

From the event yields and the ratio of efficiencies, and using the known $\phi \rightarrow K^{+} K^{-}$ branching fraction [7], the branching fraction ratio is measured to be

$$
\frac{\mathcal{B}\left(B_{s}^{0} \rightarrow J / \psi \phi \phi\right)}{\mathcal{B}\left(B_{s}^{0} \rightarrow J / \psi \phi\right)}=0.0115 \pm 0.0012(\text { stat }){ }_{-0.0009}^{+0.0005} \text { (syst) }
$$

The systematic uncertainty will be discussed in section 5 .

From the fit to the $B_{s}^{0}$ invariant mass distribution in the $B_{s}^{0} \rightarrow J / \psi \phi \phi$ decay, the mass of the $B_{s}^{0}$ meson is measured to be

$$
m\left(B_{s}^{0}\right)=5367.08 \pm 0.38 \text { (stat) } \pm 0.15 \text { (syst) } \mathrm{MeV} / c^{2} .
$$

The $J / \psi \phi$ and $\phi \phi$ mass distributions are shown in figure 3 for both data and simulation. For the data, the sPlot technique is used to subtract the background from the signal. Since the $B_{s}^{0} \rightarrow J / \psi \phi \phi$ process is a decay of a pseudoscalar into three vector mesons, its accurate description is complex and affected by large theoretical uncertainty. Here, to simulate the $B_{s}^{0} \rightarrow J / \psi \phi \phi$ decay, a simple phase-space decay model is used, which turns out not to provide a satisfactory description of the data. The disagreement can be due to either intermediate resonances or the simplified description of the decay. More data are needed to resolve the issue. Presently, due to the low statistics and the unknown decay dynamics, it is difficult to draw any conclusions from the two mass distributions.

\section{Systematic uncertainties}

A summary of the systematic uncertainties on the measurement of the branching fraction ratio is given in table 1 . Since the various effects are uncorrelated, the total systematic uncertainty is evaluated by adding all terms in quadrature.

The average multiplicity of $B_{s}^{0} \rightarrow J / \psi \phi \phi$ candidates in the simulated sample is 1.006 compared to 1.018 of the data. The relative difference $(1.2 \%)$ is assigned as a systematic uncertainty. 

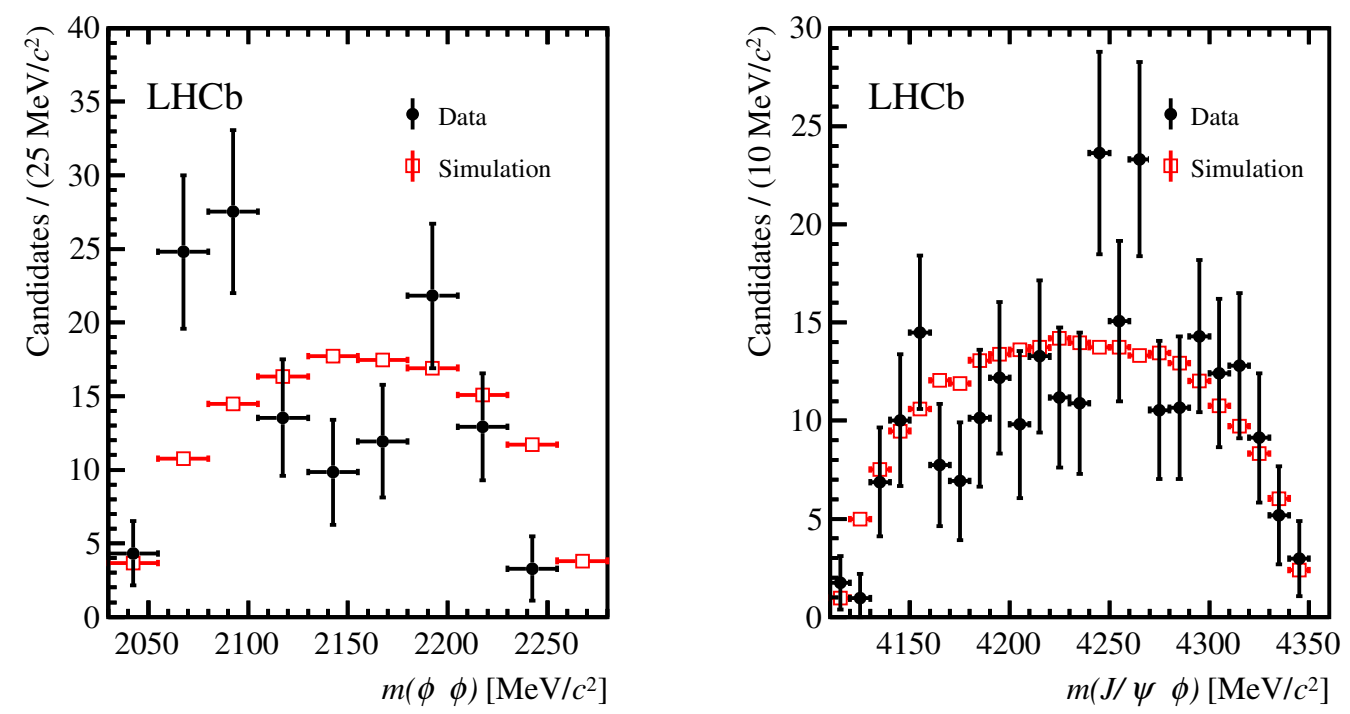

Figure 3. Invariant mass of the $(\phi, \phi)$ (left) and $(J / \psi, \phi)$ (right) pairs, in the $B_{s}^{0} \rightarrow J / \psi \phi \phi$ decay. In the $J / \psi \phi$ plot, for each candidate two values are calculated, one for each $\phi$ meson. The distribution of data and simulation (phase space) are shown. To compare the shape, the two distributions are normalised to the same area.

The use of a simplified decay model affects the determination of the detection efficiency and introduces some bias in the measurement. In order to evaluate the effect, the simulated sample is used to study the efficiency of the selection as a function of the two masses $m(\phi, \phi)$ and $m(J / \psi, \phi)$. The efficiency is then evaluated in a simulated sample reweighted in such a way as to reproduce the mass distributions in the data. A relative difference $\Delta \epsilon / \epsilon=1.0 \%$ is found and is assigned as a systematic uncertainty due to the unknown decay model.

Alternative functions for describing the signal component are tested: double Gaussian or double Crystal Ball function for the $B_{s}^{0} \rightarrow J / \psi \phi$ and single Gaussian or single Crystal Ball function for $B_{s}^{0} \rightarrow J / \psi \phi \phi$. In both cases a negligible change in yields is observed and therefore no systematic uncertainty is assigned. Conversely, different choices of the background parametrisation in the $B_{s}^{0} \rightarrow J / \psi \phi$ data can lead to sizeable difference in the results. In order to estimate a systematic uncertainty, the fits are repeated using an exponential, a second-order polynomial and the sum of the two (the nominal fit). The largest difference in yield, $1.6 \%$, between the nominal fit and the fit with the exponential, is taken as the systematic uncertainty. The same procedure applied to the signal channel results in a $0.8 \%$ change in yield. The two systematic uncertainties are added in quadrature to give an overall uncertainty of $1.8 \%$ in the modelling of the signal and backgrounds.

To evaluate the contamination from non-resonant $B_{s}^{0} \rightarrow J / \psi \phi K^{+} K^{-}$and $B_{s}^{0} \rightarrow J / \psi K^{+} K^{-} K^{+} K^{-}$decays, a dedicated search is performed for these two channels in the whole allowed kinematic region (without any requirement on the $K^{+} K^{-}$mass). The yields are then extrapolated to the restricted kinematic region of the signal. For the $B_{s}^{0} \rightarrow J / \psi K^{+} K^{-} K^{+} K^{-}$decay, the $s P l o t$ technique is first used to select the $B_{s}^{0}$ decay and then the two $K^{+} K^{-}$mass spectra are fitted simultaneously to determine the yield of the 
fully resonant decay candidates and the non-resonant ones. The non-resonant component is the sum of true non-resonant decays plus the candidates obtained by exchanging the kaons pairings in the resonant decays. When the latter component is subtracted from the measured yield, the number of non-resonant candidates is found to be $22 \pm 18$. Extrapolating this number to the $\phi$ meson mass region and using the Feldman-Cousins method [36] gives an upper limit of 1.5 events in the signal region at $68.3 \%$ confidence level. A similar procedure is followed for the $B_{s}^{0} \rightarrow J / \psi \phi K^{+} K^{-}$decay. One $K^{+} K^{-}$pair is required to have the mass in the non-resonant range, $m\left(K^{+} K^{-}\right)>1080 \mathrm{MeV} / c^{2}$. In these events, no evidence of a mass peak is found in the mass spectrum $m\left(J / \psi K^{+} K^{-} K^{+} K^{-}\right)$nor in the mass spectrum of the other kaon pair. Using the Feldman-Cousins method an estimated contamination of 6.2 events is found at $68.3 \%$ confidence level. The uncertainties on the two non-resonant modes are added linearly, resulting in an asymmetric relative uncertainty of $-6 \%$.

The data-driven method used to correct the tracking efficiency for the two additional kaons in the final state of $B_{s}^{0} \rightarrow J / \psi \phi \phi$ with respect to $B_{s}^{0} \rightarrow J / \psi \phi$ decay has an uncertainty of $1.5 \%$ per track, resulting in an overall relative uncertainty of $3.0 \%$. This term also takes into account the uncertainty of hadronic interactions in the detector material.

Due to the decay time requirement on the selected events, the lack of knowledge of the admixture of $B_{s H}^{0}$ and $B_{s L}^{0}$ eigenstates in the $B_{s}^{0} \rightarrow J / \psi \phi \phi$ decay is a further source of systematic uncertainty [37]. While for the $B_{s}^{0} \rightarrow J / \psi \phi$ decay the simulation uses the measured fractions of $B_{s H}^{0}$ and $B_{s L}^{0}$ states [38], the $B_{s}^{0} \rightarrow J / \psi \phi \phi$ decay is simulated assuming a completely symmetric combination. In order to evaluate the systematic effect, the simulated sample is reweighted assuming the two extreme cases where the $B_{s}$ meson is a complete $B_{s H}^{0}$ or a $B_{s L}^{0}$ state. The observed difference in the efficiency is $2.1 \%$ and this number is assigned as the systematic uncertainty.

A detailed comparison between data and simulation is performed for all the variables used in the BDT selection. For both the $B_{s}^{0} \rightarrow J / \psi \phi \phi$ and $B_{s}^{0} \rightarrow J / \psi \phi$ decay channels, all variables show good agreement and the relative branching fraction result is stable against changes in the threshold of the BDT response. The total systematic uncertainty on the ratio of branching fractions is found to be ${ }_{-7.4}^{+4.4} \%$.

Table 2 gives a summary of the systematic uncertainties of the $B_{s}^{0}$ mass measurement. For the $B_{s}^{0}$ mass determination, the momentum scale calibration is the main source of systematic uncertainty. The momentum scale takes into account the limited knowledge of the detector alignment. By comparing measured mass values for several charmed mesons with precisely known values, an uncertainty of $0.03 \%$ on the momentum scale is estimated [39]. The corresponding uncertainty in the $B_{s}^{0}$ mass value is $\pm 0.12 \mathrm{MeV} / c^{2}$.

The uncertainty in the kaon mass [7] will affect the $B_{s}^{0}$ mass determination, while the uncertainty on the $J / \psi$ mass has a negligible effect. The effect is estimated by repeating the fit with the kaon mass shifted by $\pm \sigma$, where $\sigma$ is the uncertainty on the known kaon mass. The observed mass variation, $\pm 0.06 \mathrm{MeV} / c^{2}$, is assigned as a systematic uncertainty.

The fit model for the signal and background of the invariant mass distributions is another source of systematic uncertainty. The effect is estimated by comparing to the nominal case the fit results with those from alternative functions. The systematic uncertainty from this effect is $\pm 0.02 \mathrm{MeV} / c^{2}$. 


\begin{tabular}{|lc|}
\hline Source & Value \\
\hline Candidate multiplicity & \pm 1.2 \\
Decay model & \pm 1.0 \\
Signal and background modelling & \pm 1.8 \\
Contamination from non-resonant decays & -6.0 \\
Track efficiency & \pm 3.0 \\
$B_{s H}^{0} / B_{s L}^{0}$ fractions & \pm 2.1 \\
\hline Total & ${ }_{-7.4}^{+4.4}$ \\
\hline
\end{tabular}

Table 1. Summary of the relative systematic uncertainties (in percentage) on the branching fraction ratio measurement.

\begin{tabular}{|lc|}
\hline Source & Value \\
\hline Momentum scale & 0.12 \\
Kaon mass & 0.06 \\
Kaon energy loss & 0.06 \\
Signal and background modelling & 0.02 \\
\hline Total & 0.15 \\
\hline
\end{tabular}

Table 2. Summary of the absolute systematic uncertainties (in $\mathrm{MeV} / \mathrm{c}^{2}$ ) affecting the $B_{s}^{0}$ mass determination from the $B_{s}^{0} \rightarrow J / \psi \phi \phi$ decay.

The energy loss of the kaons in the detector is another possible source of bias in the mass measurement. A detailed study of this effect has been performed in ref. [8] for the $B_{s}^{0} \rightarrow J / \psi \phi$ decay. Following the same procedure in $B_{s}^{0} \rightarrow J / \psi \phi \phi$ decay, the effect is found to be $\pm 0.06 \mathrm{MeV} / c^{2}$, which is assigned as a systematic uncertainty. The bias for neglecting the QED radiative corrections in the final state is negligible due to the restricted phase space [8]. The uncertainty due to detector alignment is also negligible. Combining all of the above sources in quadrature, the total systematic uncertainty on the mass measurement is found to be $\pm 0.15 \mathrm{MeV} / c^{2}$.

As a cross check, the mass measurement is performed separately in the two datataking periods and in two samples with opposite magnet polarity. All the measurements are consistent within the uncertainties.

\section{Conclusions}

This paper presents the first observation of the $B_{s}^{0} \rightarrow J / \psi \phi \phi$ decay channel, with a signal yield of $128 \pm 13$. Taking the $B_{s}^{0} \rightarrow J / \psi \phi$ decay as the reference channel the relative branching fraction is measured to be

$$
\frac{\mathcal{B}\left(B_{s} \rightarrow J / \psi \phi \phi\right)}{\mathcal{B}\left(B_{s} \rightarrow J / \psi \phi\right)}=0.0115 \pm 0.0012 \text { (stat) }{ }_{-0.0009}^{+0.0005} \text { (syst) }
$$


From a fit to the $B_{s}^{0}$ invariant mass distribution in the $B_{s}^{0} \rightarrow J / \psi \phi \phi$ decay, the mass of the $B_{s}^{0}$ meson is measured to be

$$
m\left(B_{s}^{0}\right)=5367.08 \pm 0.38 \text { (stat) } \pm 0.15 \text { (syst) } \mathrm{MeV} / c^{2} .
$$

This value is consistent with previous LHCb results [8] and with the world average [7]. The overall uncertainty is $20 \%$ larger than the current most precise measurement. As the systematic uncertainty is a factor of two smaller, further improvement can be expected when larger datasets become available.

\section{Acknowledgments}

We express our gratitude to our colleagues in the CERN accelerator departments for the excellent performance of the LHC. We thank the technical and administrative staff at the LHCb institutes. We acknowledge support from CERN and from the national agencies: CAPES, CNPq, FAPERJ and FINEP (Brazil); NSFC (China); CNRS/IN2P3 (France); BMBF, DFG and MPG (Germany); INFN (Italy); FOM and NWO (The Netherlands); MNiSW and NCN (Poland); MEN/IFA (Romania); MinES and FANO (Russia); MinECo (Spain); SNSF and SER (Switzerland); NASU (Ukraine); STFC (United Kingdom); NSF (U.S.A.). We acknowledge the computing resources that are provided by CERN, IN2P3 (France), KIT and DESY (Germany), INFN (Italy), SURF (The Netherlands), PIC (Spain), GridPP (United Kingdom), RRCKI and Yandex LLC (Russia), CSCS (Switzerland), IFIN-HH (Romania), CBPF (Brazil), PL-GRID (Poland) and OSC (U.S.A.). We are indebted to the communities behind the multiple open source software packages on which we depend. Individual groups or members have received support from AvH Foundation (Germany), EPLANET, Marie Skłodowska-Curie Actions and ERC (European Union), Conseil Général de Haute-Savoie, Labex ENIGMASS and OCEVU, Région Auvergne (France), RFBR and Yandex LLC (Russia), GVA, XuntaGal and GENCAT (Spain), The Royal Society, Royal Commission for the Exhibition of 1851 and the Leverhulme Trust (United Kingdom).

Open Access. This article is distributed under the terms of the Creative Commons Attribution License (CC-BY 4.0), which permits any use, distribution and reproduction in any medium, provided the original author(s) and source are credited.

\section{References}

[1] BABAR collaboration, J.P. Lees et al., Study of $B^{ \pm, 0} \rightarrow J / \psi K^{+} K^{-} K^{ \pm, 0}$ and search for $B^{0} \rightarrow J / \psi \phi$ at BABAR, Phys. Rev. D 91 (2015) 012003 [arXiv: 1407.7244] [INSPIRE].

[2] LHCb collaboration, Search for the $X(4140)$ state in $B^{+} \rightarrow J / \psi \phi K^{+}$decays, Phys. Rev. D 85 (2012) 091103 [arXiv: 1202.5087] [INSPIRE].

[3] CDF collaboration, T. Aaltonen et al., Evidence for a narrow near-threshold structure in the $J / \psi \phi$ mass spectrum in $B^{+} \rightarrow J / \psi \phi K^{+}$decays, Phys. Rev. Lett. 102 (2009) 242002 [arXiv:0903.2229] [INSPIRE]. 
[4] E.B. Gregory et al., Precise $B, B_{s}$ and $B_{c}$ meson spectroscopy from full lattice $Q C D$, Phys. Rev. D 83 (2011) 014506 [arXiv: 1010.3848] [INSPIRE].

[5] C. McNeile, C.T.H. Davies, E. Follana, K. Hornbostel and G.P. Lepage, Heavy meson masses and decay constants from relativistic heavy quarks in full lattice QCD, Phys. Rev. D 86 (2012) 074503 [arXiv:1207.0994] [INSPIRE].

[6] R. Lewis and R.M. Woloshyn, Bottom baryons from a dynamical lattice QCD simulation, Phys. Rev. D 79 (2009) 014502 [arXiv:0806.4783] [inSPIRE].

[7] Particle Data Group collaboration, K.A. Olive et al., Review of particle physics, Chin. Phys. C 38 (2014) 090001 [INSPIRE].

[8] LHCb collaboration, Measurement of b-hadron masses, Phys. Lett. B 708 (2012) 241 [arXiv:1112.4896] [INSPIRE].

[9] E.S. Swanson, The new heavy mesons: a status report, Phys. Rept. 429 (2006) 243 [hep-ph/0601110] [INSPIRE].

[10] E. Klempt and A. Zaitsev, Glueballs, hybrids, multiquarks. experimental facts versus $Q C D$ inspired concepts, Phys. Rept. 454 (2007) 1 [arXiv:0708.4016] [INSPIRE].

[11] BELle collaboration, C.P. Shen et al., Evidence for a new resonance and search for the $Y(4140)$ in the $\gamma \gamma \rightarrow \phi J / \psi$ process, Phys. Rev. Lett. 104 (2010) 112004 [arXiv:0912.2383] [INSPIRE].

[12] D0 collaboration, V.M. Abazov et al., Search for the $X(4140)$ state in $B^{+} \rightarrow J / \psi \phi K^{+}$decay with the Do detector Phys. Rev. D 89 (2014) 012004 [arXiv: 1309.6580] [InSPIRE].

[13] CMS collaboration, Observation of a peaking structure in the $J / \psi \phi$ mass spectrum from $B^{ \pm} \rightarrow J / \psi \phi K^{ \pm}$decays, Phys. Lett. B 734 (2014) 261 [arXiv:1309.6920] [INSPIRE].

[14] BES collaboration, M. Ablikim et al., Partial wave analysis of $J / \psi \rightarrow \gamma \phi \phi$, Phys. Lett. B 662 (2008) 330 [arXiv:0801.3885] [INSPIRE].

[15] MARK-III collaboration, Z. Bai et al., Observation of a pseudoscalar state in $J / \psi \rightarrow \gamma \phi \phi$ near $\phi \phi$ threshold, Phys. Rev. Lett. 65 (1990) 1309 [INSPIRE].

[16] DM2 collaboration, D. Bisello et al., Search of glueballs in the $J / \psi \rightarrow \gamma \phi \phi$ decay, Phys. Lett. B 179 (1986) 294 [INSPIRE].

[17] BABAR collaboration, B. Aubert et al., Rare B decays into states containing a $J / \psi$ meson

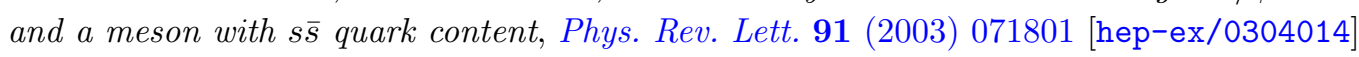
[INSPIRE].

[18] LHCb collaboration, The LHCb detector at the LHC, 2008 JINST 3 S08005 [INSPIRE].

[19] LHCb collaboration, LHCb detector performance, Int. J. Mod. Phys. A 30 (2015) 1530022 [arXiv: 1412.6352] [INSPIRE].

[20] T. Sjöstrand, S. Mrenna and P.Z. Skands, PYTHIA 6.4 physics and manual, JHEP 05 (2006) 026 [hep-ph/0603175] [INSPIRE].

[21] LHCb collaboration, Handling of the generation of primary events in Gauss, the LHCb simulation framework, J. Phys. Conf. Ser. 331 (2011) 032047 [InSPIRE].

[22] D.J. Lange, The EvtGen particle decay simulation package, Nucl. Instrum. Meth. A 462 (2001) 152 [INSPIRE]. 
[23] P. Golonka and Z. Was, PHOTOS Monte Carlo: a precision tool for QED corrections in Z and $W$ decays, Eur. Phys. J. C 45 (2006) 97 [hep-ph/0506026] [INSPIRE].

[24] GEANT4 collaboration, J. Allison et al., GEANT4 developments and applications, IEEE Trans. Nucl. Sci. 53 (2006) 270.

[25] GEANT4 collaboration, S. Agostinelli et al., GEANT4: a simulation toolkit, Nucl. Instrum. Meth. A 506 (2003) 250 [INSPIRE].

[26] LHCb collaboration, The LHCb simulation application, Gauss: design, evolution and experience, J. Phys. Conf. Ser. 331 (2011) 032023 [INSPIRE].

[27] W.D. Hulsbergen, Decay chain fitting with a Kalman filter, Nucl. Instrum. Meth. A 552 (2005) 566 [physics/0503191] [INSPIRE].

[28] L. Breiman, J.H. Friedman, R.A. Olshen and C.J. Stone, Classification and regression trees, Wadsworth international group, Belmont, California U.S.A. (1984).

[29] B.P. Roe, H.-J. Yang, J. Zhu, Y. Liu, I. Stancu and G. McGregor, Boosted decision trees, an alternative to artificial neural networks, Nucl. Instrum. Meth. A 543 (2005) 577 [physics/0408124] [INSPIRE].

[30] R. E. Schapire and Y. Freund, A decision-theoretic generalization of on-line learning and an application to boosting, J. Comput. Syst. Sci. 55 (1997) 119.

[31] G. Punzi, Sensitivity of searches for new signals and its optimization, eConf C 030908 (2003) MODT002 [physics/0308063] [INSPIRE].

[32] S. S. Wilks, The large-sample distribution of the likelihood ratio for testing composite hypotheses, Ann. Math. Stat. 9 (1938) 60.

[33] M. Pivk and F.R. Le Diberder, SPlot: a statistical tool to unfold data distributions, Nucl. Instrum. Meth. A 555 (2005) 356 [physics/0402083] [INSPIRE].

[34] T. Skwarnicki, A study of the radiative cascade transitions between the $\Upsilon^{\prime}$ and $\Upsilon$ resonances, Ph.D. thesis, Institute of Nuclear Physics, Krakow, Poland (1986) [DESY-F31-86-02] [INSPIRE].

[35] LHCb collaboration, Measurement of the track reconstruction efficiency at LHCb, 2015 JINST 10 P02007 [arXiv: 1408.1251] [INSPIRE].

[36] G.J. Feldman and R.D. Cousins, A unified approach to the classical statistical analysis of small signals, Phys. Rev. D 57 (1998) 3873 [physics/9711021] [InSPIRE].

[37] K. De Bruyn, R. Fleischer, R. Knegjens, P. Koppenburg, M. Merk and N. Tuning, Branching ratio measurements of $B_{s}$ decays, Phys. Rev. D 86 (2012) 014027 [arXiv:1204.1735] [INSPIRE].

[38] LHCb collaboration, Precision measurement of $C P$ violation in $B_{s}^{0} \rightarrow J / \psi K^{+} K^{-}$decays, Phys. Rev. Lett. 114 (2015) 041801 [arXiv:1411.3104] [INSPIRE].

[39] LHCb collaboration, Precision measurement of D meson mass differences, JHEP 06 (2013) 065 [arXiv: 1304.6865] [inSPIRE]. 


\section{The LHCb collaboration}

R. Aaij ${ }^{38}$, B. Adeva ${ }^{37}$, M. Adinolfi ${ }^{46}$, A. Affolder ${ }^{52}$, Z. Ajaltouni ${ }^{5}$, S. Akar ${ }^{6}$, J. Albrecht ${ }^{9}$, F. Alessio ${ }^{38}$, M. Alexander ${ }^{51}$, S. Ali ${ }^{41}$, G. Alkhazov ${ }^{30}$, P. Alvarez Cartelle ${ }^{53}$, A.A. Alves Jr ${ }^{57}$, S. Amato ${ }^{2}$, S. Amerio ${ }^{22}$, Y. Amhis ${ }^{7}$, L. An ${ }^{3}$, L. Anderlini ${ }^{17}$, J. Anderson ${ }^{40}$, G. Andreassi ${ }^{39}$, M. Andreotti ${ }^{16, f}$, J.E. Andrews ${ }^{58}$, R.B. Appleby ${ }^{54}$, O. Aquines Gutierrez ${ }^{10}$, F. Archilli ${ }^{38}$, P. d'Argent ${ }^{11}$, A. Artamonov ${ }^{35}$, M. Artuso ${ }^{59}$, E. Aslanides ${ }^{6}$, G. Auriemma ${ }^{25, m}$, M. Baalouch ${ }^{5}$, S. Bachmann ${ }^{11}$, J.J. Back ${ }^{48}$, A. Badalov ${ }^{36}$, C. Baesso ${ }^{60}$, W. Baldini ${ }^{16,38}$, R.J. Barlow ${ }^{54}$, C. Barschel $^{38}$, S. Barsuk ${ }^{7}$, W. Barter ${ }^{38}$, V. Batozskaya ${ }^{28}$, V. Battista ${ }^{39}$, A. Bay ${ }^{39}$, L. Beaucourt ${ }^{4}$, J. Beddow ${ }^{51}$, F. Bedeschi' ${ }^{23}$, I. Bediaga ${ }^{1}$, L.J. Bel ${ }^{41}$, V. Bellee ${ }^{39}$, N. Belloli ${ }^{20}$, I. Belyaev ${ }^{31}$, E. Ben-Haim ${ }^{8}$, G. Bencivenni ${ }^{18}$, S. Benson ${ }^{38}$, J. Benton ${ }^{46}$, A. Berezhnoy ${ }^{32}$, R. Bernet ${ }^{40}$, A. Bertolin ${ }^{22}$, M.-O. Bettler ${ }^{38}$, M. van Beuzekom ${ }^{41}$, A. Bien ${ }^{11}$, S. Bifani ${ }^{45}$, P. Billoir ${ }^{8}$, T. Bird ${ }^{54}$, A. Birnkraut ${ }^{9}$, A. Bizzeti ${ }^{17, h}$, T. Blake ${ }^{48}$, F. Blanc ${ }^{39}$, J. Blouw ${ }^{10}$, S. Blusk ${ }^{59}$, V. Bocci ${ }^{25}$, A. Bondar ${ }^{34}$, N. Bondar ${ }^{30,38}$, W. Bonivento ${ }^{15}$, S. Borghi ${ }^{54}$, M. Borsato ${ }^{7}$, T.J.V. Bowcock ${ }^{52}$, E. Bowen ${ }^{40}$, C. Bozzi ${ }^{16}$, S. Braun ${ }^{11}$, M. Britsch ${ }^{10}$, T. Britton ${ }^{59}$, J. Brodzicka ${ }^{54}$, N.H. Brook ${ }^{46}$, A. Bursche ${ }^{40}$, J. Buytaert ${ }^{38}$, S. Cadeddu ${ }^{15}$, R. Calabrese ${ }^{16, f}$, M. Calvi ${ }^{20, j}$, M. Calvo Gomez ${ }^{36, o}$, P. Campana ${ }^{18}$, D. Campora Perez ${ }^{38}$, L. Capriotti ${ }^{54}$, A. Carbone ${ }^{14, d}$, G. Carboni ${ }^{24, k}$, R. Cardinale ${ }^{19, i}$, A. Cardini ${ }^{15}$, P. Carniti ${ }^{20}$, L. Carson $^{50}$, K. Carvalho Akiba ${ }^{2,38}$, G. Casse ${ }^{52}$, L. Cassina ${ }^{20, j}$, L. Castillo Garcia ${ }^{38}$, M. Cattaneo ${ }^{38}$, Ch. Cauet ${ }^{9}$, G. Cavallero ${ }^{19}$, R. Cenci ${ }^{23, s}$, M. Charles ${ }^{8}$, Ph. Charpentier ${ }^{38}$, M. Chefdeville ${ }^{4}$, S. Chen ${ }^{54}$, S.-F. Cheung ${ }^{55}$, N. Chiapolini ${ }^{40}$, M. Chrzaszcz ${ }^{40}$, X. Cid Vidal ${ }^{38}$, G. Ciezarek $^{41}$, P.E.L. Clarke ${ }^{50}$, M. Clemencic ${ }^{38}$, H.V. Cliff ${ }^{47}$, J. Closier ${ }^{38}$, V. Coco ${ }^{38}$, J. Cogan ${ }^{6}$, E. Cogneras ${ }^{5}$, V. Cogoni ${ }^{15, e}$, L. Cojocariu ${ }^{29}$, G. Collazuol ${ }^{22}$, P. Collins ${ }^{38}$, A. Comerma-Montells ${ }^{11}$, A. Contu ${ }^{15,38}$, A. Cook $^{46}$, M. Coombes ${ }^{46}$, S. Coquereau ${ }^{8}$, G. Corti $^{38}$, M. Corvo ${ }^{16, f}$, B. Couturier ${ }^{38}$, G.A. Cowan ${ }^{50}$, D.C. Craik ${ }^{48}$, A. Crocombe ${ }^{48}$, M. Cruz Torres ${ }^{60}$, S. Cunliffe ${ }^{53}$, R. Currie ${ }^{53}$, C. D’Ambrosio ${ }^{38}$, E. Dall'Occo ${ }^{41}$, J. Dalseno ${ }^{46}$, P.N.Y. David ${ }^{41}$, A. Davis ${ }^{57}$, K. De Bruyn ${ }^{41}$, S. De Capua ${ }^{54}$, M. De Cian ${ }^{11}$, J.M. De Miranda ${ }^{1}$, L. De Paula ${ }^{2}$, P. De Simone ${ }^{18}$, C.-T. Dean ${ }^{51}$, D. Decamp ${ }^{4}$, M. Deckenhoff ${ }^{9}$, L. Del Buono ${ }^{8}$, N. Déléage ${ }^{4}$, M. Demmer ${ }^{9}$, D. Derkach ${ }^{55}$, O. Deschamps ${ }^{5}$, F. Dettori ${ }^{38}$, B. Dey ${ }^{21}$, A. Di Canto ${ }^{38}$, F. Di Ruscio ${ }^{24}$, H. Dijkstra ${ }^{38}$, S. Donleavy ${ }^{52}$, F. Dordei ${ }^{11}$, M. Dorigo ${ }^{39}$, A. Dosil Suárez ${ }^{37}$, D. Dossett ${ }^{48}$, A. Dovbnya ${ }^{43}$, K. Dreimanis ${ }^{52}$, L. Dufour ${ }^{41}$, G. Dujany ${ }^{54}$, F. Dupertuis ${ }^{39}$, P. Durante ${ }^{38}$, R. Dzhelyadin ${ }^{35}$, A. Dziurda ${ }^{26}$, A. Dzyuba ${ }^{30}$, S. Easo ${ }^{49,38}$, U. Egede ${ }^{53}$, V. Egorychev ${ }^{31}$, S. Eidelman ${ }^{34}$, S. Eisenhardt ${ }^{50}$, U. Eitschberger ${ }^{9}$, R. Ekelhof ${ }^{9}$, L. Eklund ${ }^{51}$, I. El Rifai ${ }^{5}$, Ch. Elsasser ${ }^{40}$, S. Ely ${ }^{59}$, S. Esen ${ }^{11}$, H.M. Evans ${ }^{47}$, T. Evans ${ }^{55}$, A. Falabella ${ }^{14}$, C. Färber ${ }^{38}$, N. Farley ${ }^{45}$, S. Farry ${ }^{52}$, R. Fay ${ }^{52}$, D. Ferguson ${ }^{50}$, V. Fernandez Albor ${ }^{37}$, F. Ferrari ${ }^{14}$, F. Ferreira Rodrigues ${ }^{1}$, M. Ferro-Luzzi ${ }^{38}$, S. Filippov ${ }^{33}$, M. Fiore ${ }^{16,38, f}$, M. Fiorini ${ }^{16, f}$,

M. Firlej ${ }^{27}$, C. Fitzpatrick ${ }^{39}$, T. Fiutowski ${ }^{27}$, K. Fohl $^{38}$, P. Fol $^{53}$, M. Fontana ${ }^{15}$, F. Fontanelli ${ }^{19, i}$, R. Forty ${ }^{38}$, O. Francisco ${ }^{2}$, M. Frank ${ }^{38}$, C. Frei ${ }^{38}$, M. Frosini ${ }^{17}$, J. Fu ${ }^{21}$, E. Furfaro ${ }^{24, k}$,

A. Gallas Torreira ${ }^{37}$, D. Galli ${ }^{14, d}$, S. Gallorini ${ }^{22,38}$, S. Gambetta ${ }^{50}$, M. Gandelman ${ }^{2}$, P. Gandini ${ }^{55}$, Y. Gao ${ }^{3}$, J. García Pardiñas ${ }^{37}$, J. Garra Tico ${ }^{47}$, L. Garrido ${ }^{36}$, D. Gascon ${ }^{36}$, C. Gaspar ${ }^{38}$,

R. Gauld ${ }^{55}$, L. Gavardi ${ }^{9}$, G. Gazzoni ${ }^{5}$, A. Geraci ${ }^{21, u}$, D. Gerick ${ }^{11}$, E. Gersabeck ${ }^{11}$,

M. Gersabeck ${ }^{54}$, T. Gershon ${ }^{48}$, Ph. Ghez ${ }^{4}$, A. Gianelle ${ }^{22}$, S. Gianì ${ }^{39}$, V. Gibson ${ }^{47}$, O. G. Girard ${ }^{39}$, L. Giubega ${ }^{29}$, V.V. Gligorov ${ }^{38}$, C. Göbel ${ }^{60}$, D. Golubkov ${ }^{31}$, A. Golutvin ${ }^{53,31,38}$, A. Gomes ${ }^{1, a}$,

C. Gotti ${ }^{20, j}$, M. Grabalosa Gándara ${ }^{5}$, R. Graciani Diaz ${ }^{36}$, L.A. Granado Cardoso ${ }^{38}$, E. Graugés ${ }^{36}$, E. Graverini ${ }^{40}$, G. Graziani ${ }^{17}$, A. Grecu ${ }^{29}$, E. Greening ${ }^{55}$, S. Gregson ${ }^{47}$, P. Griffith ${ }^{45}$, L. Grillo ${ }^{11}$, O. Grünberg ${ }^{63}$, B. Gui ${ }^{59}$, E. Gushchin ${ }^{33}$, Yu. Guz ${ }^{35,38}$, T. Gys ${ }^{38}$, T. Hadavizadeh ${ }^{55}$, C. Hadjivasiliou ${ }^{59}$, G. Haefeli ${ }^{39}$, C. Haen ${ }^{38}$, S.C. Haines ${ }^{47}$, S. Hall ${ }^{53}$, B. Hamilton ${ }^{58}$, X. Han ${ }^{11}$,

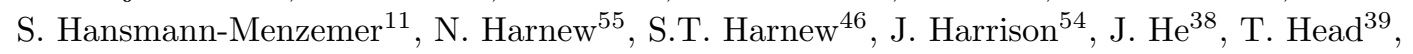
V. Heijne ${ }^{41}$, K. Hennessy ${ }^{52}$, P. Henrard ${ }^{5}$, L. Henry ${ }^{8}$, J.A. Hernando Morata ${ }^{37}$, 
E. van Herwijnen ${ }^{38}$, M. Heß ${ }^{63}$, A. Hicheur ${ }^{2}$, D. Hill ${ }^{55}$, M. Hoballah ${ }^{5}$, C. Hombach ${ }^{54}$, W. Hulsbergen ${ }^{41}$, T. Humair ${ }^{53}$, N. Hussain ${ }^{55}$, D. Hutchcroft ${ }^{52}$, D. Hynds ${ }^{51}$, M. Idzik ${ }^{27}$, P. Ilten ${ }^{56}$, R. Jacobsson ${ }^{38}$, A. Jaeger ${ }^{11}$, J. Jalocha ${ }^{55}$, E. Jans ${ }^{41}$, A. Jawahery ${ }^{58}$, F. Jing ${ }^{3}$, M. John ${ }^{55}$, D. Johnson ${ }^{38}$, C.R. Jones ${ }^{47}$, C. Joram ${ }^{38}$, B. Jost ${ }^{38}$, N. Jurik ${ }^{59}$, S. Kandybei ${ }^{43}$, W. Kanso ${ }^{6}$, M. Karacson ${ }^{38}$, T.M. Karbach ${ }^{38, \dagger}$, S. Karodia ${ }^{51}$, M. Kelsey ${ }^{59}$, I.R. Kenyon ${ }^{45}$, M. Kenzie ${ }^{38}$, T. Ketel ${ }^{42}$, B. Khanji ${ }^{20,38, j}$, C. Khurewathanakul ${ }^{39}$, S. Klaver ${ }^{54}$, K. Klimaszewski ${ }^{28}$, O. Kochebina ${ }^{7}$, M. Kolpin ${ }^{11}$, I. Komarov ${ }^{39}$, R.F. Koopman ${ }^{42}$, P. Koppenburg ${ }^{41,38}$, M. Kozeiha ${ }^{5}$, L. Kravchuk ${ }^{33}$, K. Kreplin ${ }^{11}$, M. Kreps ${ }^{48}$, G. Krocker ${ }^{11}$, P. Krokovny ${ }^{34}$, F. Kruse ${ }^{9}$, W. Krzemien ${ }^{28}$, W. Kucewicz ${ }^{26, n}$, M. Kucharczyk ${ }^{26}$, V. Kudryavtsev ${ }^{34}$, A. K. Kuonen ${ }^{39}$, K. Kurek ${ }^{28}$, T. Kvaratskheliya ${ }^{31}$, D. Lacarrere ${ }^{38}$, G. Lafferty ${ }^{54}$, A. Lai ${ }^{15}$, D. Lambert ${ }^{50}$, G. Lanfranchi ${ }^{18}$, C. Langenbruch ${ }^{48}$, B. Langhans ${ }^{38}$, T. Latham ${ }^{48}$, C. Lazzeroni ${ }^{45}$, R. Le Gac ${ }^{6}$, J. van Leerdam ${ }^{41}$, J.-P. Lees ${ }^{4}$, R. Lefèvre ${ }^{5}$, A. Leflat ${ }^{32,38}$, J. Lefrançois ${ }^{7}$, E. Lemos Cid $^{37}$,

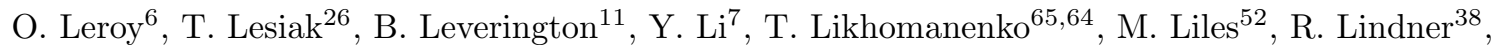

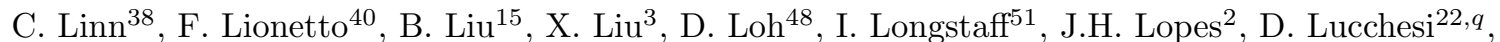
M. Lucio Martinez ${ }^{37}$, H. Luo ${ }^{50}$, A. Lupato ${ }^{22}$, E. Luppi ${ }^{16, f}$, O. Lupton ${ }^{55}$, N. Lusardi ${ }^{21}$,

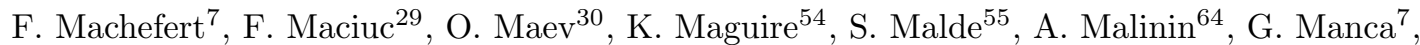
G. Mancinelli ${ }^{6}$, P. Manning ${ }^{59}$, A. Mapelli ${ }^{38}$, J. Maratas ${ }^{5}$, J.F. Marchand ${ }^{4}$, U. Marconi ${ }^{14}$, C. Marin Benito ${ }^{36}$, P. Marino ${ }^{23,38, s}$, J. Marks ${ }^{11}$, G. Martellotti ${ }^{25}$, M. Martin ${ }^{6}$, M. Martinelli ${ }^{39}$, D. Martinez Santos ${ }^{37}$, F. Martinez Vidal ${ }^{66}$, D. Martins Tostes ${ }^{2}$, A. Massafferri ${ }^{1}$, R. Matev ${ }^{38}$, A. Mathad ${ }^{48}$, Z. Mathe ${ }^{38}$, C. Matteuzzi ${ }^{20}$, K. Matthieu ${ }^{11}$, A. Mauri ${ }^{40}$, B. Maurin ${ }^{39}$, A. Mazurov ${ }^{45}$, M. McCann ${ }^{53}$, J. McCarthy ${ }^{45}$, A. $\mathrm{McNab}^{54}$, R. McNulty ${ }^{12}$, B. Meadows ${ }^{57}$, F. Meier ${ }^{9}$, M. Meissner ${ }^{11}$, D. Melnychuk ${ }^{28}$, M. Merk ${ }^{41}$, D.A. Milanes ${ }^{62}$, M.-N. Minard ${ }^{4}$, D.S. Mitzel ${ }^{11}$, J. Molina Rodriguez ${ }^{60}$, I.A. Monroy ${ }^{62}$, S. Monteil ${ }^{5}$, M. Morandin ${ }^{22}$, P. Morawski ${ }^{27}$, A. Mordà ${ }^{6}$, M.J. Morello ${ }^{23, s}$, J. Moron ${ }^{27}$, A.B. Morris ${ }^{50}$, R. Mountain ${ }^{59}$, F. Muheim ${ }^{50}$, J. Müller ${ }^{9}$, K. Müller ${ }^{40}$, V. Müller ${ }^{9}$, M. Mussini ${ }^{14}$, B. Muster ${ }^{39}$, P. Naik ${ }^{46}$, T. Nakada ${ }^{39}$, R. Nandakumar ${ }^{49}$, A. Nandi ${ }^{55}$, I. Nasteva ${ }^{2}$, M. Needham ${ }^{50}$, N. Neri ${ }^{21}$, S. Neubert ${ }^{11}$, N. Neufeld ${ }^{38}$, M. Neuner ${ }^{11}$, A.D. Nguyen ${ }^{39}$, T.D. Nguyen ${ }^{39}$, C. Nguyen-Mau ${ }^{39, p}$, V. Niess ${ }^{5}$, R. Niet ${ }^{9}$, N. Nikitin ${ }^{32}$, T. Nikodem ${ }^{11}$, D. Ninci ${ }^{23}$, A. Novoselov ${ }^{35}$, D.P. O'Hanlon ${ }^{48}$, A. Oblakowska-Mucha ${ }^{27}$, V. Obraztsov ${ }^{35}$, S. Ogilvy ${ }^{51}$, O. Okhrimenko ${ }^{44}$, R. Oldeman ${ }^{15, e}$, C.J.G. Onderwater ${ }^{67}$, B. Osorio Rodrigues ${ }^{1}$, J.M. Otalora Goicochea ${ }^{2}$, A. Otto ${ }^{38}$, P. Owen ${ }^{53}$, A. Oyanguren ${ }^{66}$, A. Palano ${ }^{13, c}$, F. Palombo ${ }^{21, t}$, M. Palutan ${ }^{18}$, J. Panman ${ }^{38}$, A. Papanestis ${ }^{49}$, M. Pappagallo ${ }^{51}$, L.L. Pappalardo ${ }^{16, f}$, C. Pappenheimer ${ }^{57}$, C. Parkes $^{54}$, G. Passaleva ${ }^{17}$, G.D. Patel ${ }^{52}$, M. Patel ${ }^{53}$, C. Patrignani ${ }^{19, i}$, A. Pearce ${ }^{54,49}$, A. Pellegrino ${ }^{41}$, G. Penso ${ }^{25, l}$, M. Pepe Altarelli ${ }^{38}$, S. Perazzini ${ }^{14, d}$, P. Perret ${ }^{5}$, L. Pescatore ${ }^{45}$, K. Petridis ${ }^{46}$, A. Petrolini ${ }^{19, i}$, M. Petruzzo ${ }^{21}$, E. Picatoste Olloqui ${ }^{36}$, B. Pietrzyk ${ }^{4}$, T. Pilař ${ }^{48}$, D. Pinci ${ }^{25}$, A. Pistone ${ }^{19}$, A. Piuccir ${ }^{11}$, S. Playfer ${ }^{50}$, M. Plo Casasus ${ }^{37}$, T. Poikela ${ }^{38}$, F. Polci ${ }^{8}$, A. Poluektov ${ }^{48,34}$, I. Polyakov ${ }^{31}$, E. Polycarpo ${ }^{2}$, A. Popov ${ }^{35}$, D. Popov ${ }^{10,38}$, B. Popovici ${ }^{29}$, C. Potterat ${ }^{2}$, E. Price ${ }^{46}$, J.D. Price ${ }^{52}$, J. Prisciandaro ${ }^{39}$, A. Pritchard ${ }^{52}$, C. Prouve ${ }^{46}$, V. Pugatch ${ }^{44}$, A. Puig Navarro ${ }^{39}$, G. Punzi ${ }^{23, r}$, W. Qian ${ }^{4}$, R. Quagliani ${ }^{7,46}$, B. Rachwal ${ }^{26}$, J.H. Rademacker ${ }^{46}$, M. Rama ${ }^{23}$, M.S. Rangel ${ }^{2}$, I. Raniuk ${ }^{43}$, N. Rauschmayr ${ }^{38}$, G. Raven ${ }^{42}$, F. Redi ${ }^{53}$, S. Reichert ${ }^{54}$, M.M. Reid ${ }^{48}$, A.C. dos Reis ${ }^{1}$, S. Ricciardi ${ }^{49}$, S. Richards ${ }^{46}$, M. Rihl ${ }^{38}$, K. Rinnert ${ }^{52}$, V. Rives Molina ${ }^{36}$, P. Robbe ${ }^{7,38}$, A.B. Rodrigues ${ }^{1}$, E. Rodrigues ${ }^{54}$, J.A. Rodriguez $\mathrm{Lopez}^{62}$, P. Rodriguez Perez ${ }^{54}$, S. Roiser ${ }^{38}$, V. Romanovsky ${ }^{35}$, A. Romero Vidal ${ }^{37}$, J. W. Ronayne ${ }^{12}$, M. Rotondo ${ }^{22}$, J. Rouvinet ${ }^{39}$, T. Ruf ${ }^{38}$, P. Ruiz Valls ${ }^{66}$, J.J. Saborido Silva ${ }^{37}$, N. Sagidova ${ }^{30}$, P. Sail ${ }^{51}$, B. Saitta ${ }^{15, e}$,

V. Salustino Guimaraes ${ }^{2}$, C. Sanchez Mayordomo ${ }^{66}$, B. Sanmartin Sedes ${ }^{37}$, R. Santacesaria ${ }^{25}$, C. Santamarina Rios ${ }^{37}$, M. Santimaria ${ }^{18}$, E. Santovetti ${ }^{24, k}$, A. Sarti ${ }^{18, l}$, C. Satriano ${ }^{25, m}$, A. Satta ${ }^{24}$, D.M. Saunders ${ }^{46}$, D. Savrina ${ }^{31,32}$, M. Schiller ${ }^{38}$, H. Schindler ${ }^{38}$, M. Schlupp ${ }^{9}$, M. Schmelling ${ }^{10}$, T. Schmelzer ${ }^{9}$, B. Schmidt ${ }^{38}$, O. Schneider ${ }^{39}$, A. Schopper ${ }^{38}$, M. Schubiger ${ }^{39}$, 
M.-H. Schune ${ }^{7}$, R. Schwemmer ${ }^{38}$, B. Sciascia ${ }^{18}$, A. Sciubba ${ }^{25, l}$, A. Semennikov ${ }^{31}$, N. Serra ${ }^{40}$, J. Serrano ${ }^{6}$, L. Sestini ${ }^{22}$, P. Seyfert ${ }^{20}$, M. Shapkin ${ }^{35}$, I. Shapoval ${ }^{16,43, f}$, Y. Shcheglov ${ }^{30}$, T. Shears ${ }^{52}$, L. Shekhtman ${ }^{34}$, V. Shevchenko ${ }^{64}$, A. Shires ${ }^{9}$, B.G. Siddi ${ }^{16}$, R. Silva Coutinho ${ }^{48}$, G. Simi ${ }^{22}$, M. Sirendi ${ }^{47}$, N. Skidmore ${ }^{46}$, I. Skillicorn ${ }^{51}$, T. Skwarnicki ${ }^{59}$, E. Smith ${ }^{55,49}$, E. Smith ${ }^{53}$, I. T. Smith ${ }^{50}$, J. Smith ${ }^{47}$, M. Smith ${ }^{54}$, H. Snoek ${ }^{41}$, M.D. Sokoloff ${ }^{57,38}$, F.J.P. Soler ${ }^{51}$, F. Soomro ${ }^{39}$, D. Souza ${ }^{46}$, B. Souza De Paula ${ }^{2}$, B. Spaan ${ }^{9}$, P. Spradlin ${ }^{51}$, S. Sridharan ${ }^{38}$, F. Stagni ${ }^{38}$, M. Stahl ${ }^{11}$, S. Stahl ${ }^{38}$, S. Stefkova ${ }^{53}$, O. Steinkamp ${ }^{40}$, O. Stenyakin ${ }^{35}$, S. Stevenson ${ }^{55}$, S. Stoica ${ }^{29}$, S. Stone ${ }^{59}$, B. Storaci ${ }^{40}$, S. Stracka ${ }^{23, s}$, M. Straticiuc ${ }^{29}$, U. Straumann ${ }^{40}$, L. Sun ${ }^{57}$, W. Sutcliffe ${ }^{53}$, K. Swientek ${ }^{27}$, S. Swientek ${ }^{9}$, V. Syropoulos ${ }^{42}$, M. Szczekowskii ${ }^{28}$, P. Szczypka ${ }^{39,38}$, T. Szumlak ${ }^{27}$, S. T'Jampens ${ }^{4}$, A. Tayduganov ${ }^{6}$, T. Tekampe ${ }^{9}$, M. Teklishyn ${ }^{7}$, G. Tellarini ${ }^{16, f}$,

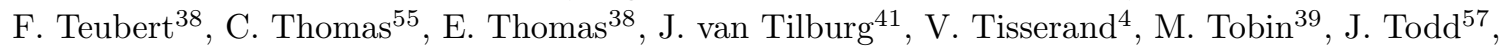
S. Tolk ${ }^{42}$, L. Tomassetti ${ }^{16, f}$, D. Tonelli ${ }^{38}$, S. Topp-Joergensen ${ }^{55}$, N. Torr ${ }^{55}$, E. Tournefier ${ }^{4}$,

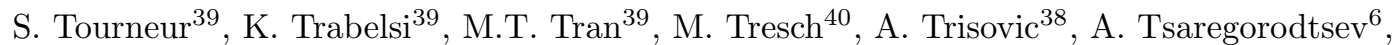
P. Tsopelas ${ }^{41}$, N. Tuning ${ }^{41,38}$, A. Ukleja ${ }^{28}$, A. Ustyuzhanin ${ }^{65,64}$, U. Uwer ${ }^{11}$, C. Vacca ${ }^{15, e}$, V. Vagnoni ${ }^{14}$, G. Valenti ${ }^{14}$, A. Vallier ${ }^{7}$, R. Vazquez Gomez ${ }^{18}$, P. Vazquez Regueiro ${ }^{37}$, C. Vázquez Sierra ${ }^{37}$, S. Vecchi ${ }^{16}$, J.J. Velthuis ${ }^{46}$, M. Veltri ${ }^{17, g}$, G. Veneziano ${ }^{39}$, M. Vesterinen ${ }^{11}$, B. Viaud ${ }^{7}$, D. Vieira ${ }^{2}$, M. Vieites Diaz ${ }^{37}$, X. Vilasis-Cardona ${ }^{36, o}$, A. Vollhardt ${ }^{40}$, D. Volyanskyy ${ }^{10}$, D. Voong ${ }^{46}$, A. Vorobyev ${ }^{30}$, V. Vorobyev ${ }^{34}$, C. Voß ${ }^{63}$, J.A. de Vries ${ }^{41}$, R. Waldi ${ }^{63}$, C. Wallace ${ }^{48}$, R. Wallace ${ }^{12}$, J. Walsh ${ }^{23}$, S. Wandernoth ${ }^{11}$, J. Wang ${ }^{59}$, D.R. Ward ${ }^{47}$, N.K. Watson ${ }^{45}$, D. Websdale ${ }^{53}$, A. Weiden ${ }^{40}$, M. Whitehead ${ }^{48}$, G. Wilkinson ${ }^{55,38}$, M. Wilkinson ${ }^{59}$, M. Williams $^{38}$, M.P. Williams ${ }^{45}$, M. Williams ${ }^{56}$, T. Williams ${ }^{45}$, F.F. Wilson ${ }^{49}$, J. Wimberley ${ }^{58}$, J. Wishahi ${ }^{9}$, W. Wislicki ${ }^{28}$, M. Witek ${ }^{26}$, G. Wormser ${ }^{7}$, S.A. Wotton ${ }^{47}$, S. Wright ${ }^{47}$, K. Wyllie $^{38}$, Y. Xie ${ }^{61}$, Z. Xu ${ }^{39}$, Z. Yang ${ }^{3}$, J. Yu ${ }^{61}$, X. Yuan ${ }^{34}$, O. Yushchenko ${ }^{35}$, M. Zangoli ${ }^{14}$, M. Zavertyaev ${ }^{10, b}$, L. Zhang ${ }^{3}$, Y. Zhang ${ }^{3}$, A. Zhelezov ${ }^{11}$, A. Zhokhov ${ }^{31}$, L. Zhong ${ }^{3}$, S. Zucchelli ${ }^{14}$

1 Centro Brasileiro de Pesquisas Físicas (CBPF), Rio de Janeiro, Brazil

2 Universidade Federal do Rio de Janeiro (UFRJ), Rio de Janeiro, Brazil

3 Center for High Energy Physics, Tsinghua University, Beijing, China

4 LAPP, Université Savoie Mont-Blanc, CNRS/IN2P3, Annecy-Le-Vieux, France

5 Clermont Université, Université Blaise Pascal, CNRS/IN2P3, LPC, Clermont-Ferrand, France

6 CPPM, Aix-Marseille Université, CNRS/IN2P3, Marseille, France

7 LAL, Université Paris-Sud, CNRS/IN2P3, Orsay, France

8 LPNHE, Université Pierre et Marie Curie, Université Paris Diderot, CNRS/IN2P3, Paris, France

9 Fakultät Physik, Technische Universität Dortmund, Dortmund, Germany

10 Max-Planck-Institut für Kernphysik (MPIK), Heidelberg, Germany

11 Physikalisches Institut, Ruprecht-Karls-Universität Heidelberg, Heidelberg, Germany

12 School of Physics, University College Dublin, Dublin, Ireland

13 Sezione INFN di Bari, Bari, Italy

14 Sezione INFN di Bologna, Bologna, Italy

15 Sezione INFN di Cagliari, Cagliari, Italy

16 Sezione INFN di Ferrara, Ferrara, Italy

17 Sezione INFN di Firenze, Firenze, Italy

18 Laboratori Nazionali dell'INFN di Frascati, Frascati, Italy

19 Sezione INFN di Genova, Genova, Italy

20 Sezione INFN di Milano Bicocca, Milano, Italy

21 Sezione INFN di Milano, Milano, Italy

22 Sezione INFN di Padova, Padova, Italy

23 Sezione INFN di Pisa, Pisa, Italy

24 Sezione INFN di Roma Tor Vergata, Roma, Italy

25 Sezione INFN di Roma La Sapienza, Roma, Italy 
Henryk Niewodniczanski Institute of Nuclear Physics Polish Academy of Sciences, Kraków, Poland AGH - University of Science and Technology, Faculty of Physics and Applied Computer Science, Kraków, Poland

28 National Center for Nuclear Research (NCBJ), Warsaw, Poland

29 Horia Hulubei National Institute of Physics and Nuclear Engineering, Bucharest-Magurele, Romania

30 Petersburg Nuclear Physics Institute (PNPI), Gatchina, Russia

31 Institute of Theoretical and Experimental Physics (ITEP), Moscow, Russia

32 Institute of Nuclear Physics, Moscow State University (SINP MSU), Moscow, Russia

33 Institute for Nuclear Research of the Russian Academy of Sciences (INR RAN), Moscow, Russia

34 Budker Institute of Nuclear Physics (SB RAS) and Novosibirsk State University, Novosibirsk, Russia

35 Institute for High Energy Physics (IHEP), Protvino, Russia

36 Universitat de Barcelona, Barcelona, Spain

37 Universidad de Santiago de Compostela, Santiago de Compostela, Spain

38 European Organization for Nuclear Research (CERN), Geneva, Switzerland

39 Ecole Polytechnique Fédérale de Lausanne (EPFL), Lausanne, Switzerland

40 Physik-Institut, Universität Zürich, Zürich, Switzerland

41 Nikhef National Institute for Subatomic Physics, Amsterdam, The Netherlands

42 Nikhef National Institute for Subatomic Physics and VU University Amsterdam, Amsterdam, The Netherlands

43 NSC Kharkiv Institute of Physics and Technology (NSC KIPT), Kharkiv, Ukraine

44 Institute for Nuclear Research of the National Academy of Sciences (KINR), Kyiv, Ukraine

45 University of Birmingham, Birmingham, United Kingdom

46 H.H. Wills Physics Laboratory, University of Bristol, Bristol, United Kingdom

47 Cavendish Laboratory, University of Cambridge, Cambridge, United Kingdom

48 Department of Physics, University of Warwick, Coventry, United Kingdom

49 STFC Rutherford Appleton Laboratory, Didcot, United Kingdom

50 School of Physics and Astronomy, University of Edinburgh, Edinburgh, United Kingdom

51 School of Physics and Astronomy, University of Glasgow, Glasgow, United Kingdom

52 Oliver Lodge Laboratory, University of Liverpool, Liverpool, United Kingdom

53 Imperial College London, London, United Kingdom

54 School of Physics and Astronomy, University of Manchester, Manchester, United Kingdom

55 Department of Physics, University of Oxford, Oxford, United Kingdom

56 Massachusetts Institute of Technology, Cambridge, MA, United States

57 University of Cincinnati, Cincinnati, OH, United States

58 University of Maryland, College Park, MD, United States

59 Syracuse University, Syracuse, NY, United States

60 Pontifícia Universidade Católica do Rio de Janeiro (PUC-Rio), Rio de Janeiro, Brazil, associated $t o^{2}$

61 Institute of Particle Physics, Central China Normal University, Wuhan, Hubei, China, associated $t o^{3}$

62 Departamento de Fisica, Universidad Nacional de Colombia, Bogota, Colombia, associated to ${ }^{8}$

63 Institut für Physik, Universität Rostock, Rostock, Germany, associated to ${ }^{11}$

64 National Research Centre Kurchatov Institute, Moscow, Russia, associated to ${ }^{31}$

65 Yandex School of Data Analysis, Moscow, Russia, associated to 31

66 Instituto de Fisica Corpuscular (IFIC), Universitat de Valencia-CSIC, Valencia, Spain, associated $t^{36}$

67 Van Swinderen Institute, University of Groningen, Groningen, The Netherlands, associated to ${ }^{41}$

a Universidade Federal do Triângulo Mineiro (UFTM), Uberaba-MG, Brazil

${ }^{b}$ P.N. Lebedev Physical Institute, Russian Academy of Science (LPI RAS), Moscow, Russia 
c Università di Bari, Bari, Italy

d Università di Bologna, Bologna, Italy

e Università di Cagliari, Cagliari, Italy

$f$ Università di Ferrara, Ferrara, Italy

$g$ Università di Urbino, Urbino, Italy

$h$ Università di Modena e Reggio Emilia, Modena, Italy

i Università di Genova, Genova, Italy

j Università di Milano Bicocca, Milano, Italy

${ }^{k}$ Università di Roma Tor Vergata, Roma, Italy

${ }^{l}$ Università di Roma La Sapienza, Roma, Italy

$m$ Università della Basilicata, Potenza, Italy

$n$ AGH - University of Science and Technology, Faculty of Computer Science, Electronics and Telecommunications, Kraków, Poland

- LIFAELS, La Salle, Universitat Ramon Llull, Barcelona, Spain

$p$ Hanoi University of Science, Hanoi, Viet Nam

$q$ Università di Padova, Padova, Italy

$r$ Università di Pisa, Pisa, Italy

$s$ Scuola Normale Superiore, Pisa, Italy

${ }^{t}$ Università degli Studi di Milano, Milano, Italy

u Politecnico di Milano, Milano, Italy ${ }^{\dagger}$ Deceased 